\title{
Scalar dissipation rate transport conditional on flow topologies in different regimes of premixed turbulent combustion
}

\author{
Nilanjan Chakraborty ${ }^{1}$, Daniel H. Wacks ${ }^{2}$, Sebastian.Ketterl ${ }^{3}$, Markus Klein ${ }^{3 *}$, Hong G. $\operatorname{Im}^{4}$
}

${ }^{1}$ School of Engineering

University of Newcastle

Claremont Road, Newcastle

NE1 7RU, UK

Email: nilanjan.chakraboty@ncl.ac.uk

${ }^{2}$ School of Engineering and Computing Sciences,

Durham University, Lower Mountjoy, South Road, Durham,

DH1 3LE, UK

Email : daniel.h.wacks@durham.ac.uk

${ }^{3}$ Universität der Bundeswehr München, Fakultät für Luft- und Raumfahrttechnik, LRT1, Werner-Heisenberg-Weg 39, 85577 Neubiberg, Germany

Email: markus.klein@unibw.de, sebastian.ketterl@unibw.de

${ }^{4}$ Clean Combustion Research Center, King Abdullah University of Science and Technology (KAUST), Thuwal 23955-6900, Saudi Arabia

Email: hong.im@kaust.edu.sa

Colloquium 5: Turbulent Flames (Turbulent-Premixed)

Running title: Scalar dissipation rate transport conditional on flow topologies

Proc. Combust. Inst. 37, 2018, Abstract: 258

Word count (MS word): TOTAL: 6198.36 (MS-Word)

Words of text: 3185

Figures: 2210.8

Tables: 136.8

Equations: 106.4

References: 559.36

\footnotetext{
${ }^{*}$ Corresponding author
} 


\section{ABSTRACT}

The Favre-averaged scalar dissipation rate transport conditional on local flow topologies in premixed turbulent flames has been analysed based on a detailed chemistry Direct Numerical Simulation database of statistically planar turbulent hydrogen-air premixed flames with an equivalence ratio of 0.7 representing the corrugated flamelets, thin reaction zones and broken reaction zones regimes of premixed turbulent combustion. The local flow topologies have been categorised by the values of the three invariants of the velocity gradient tensor and the statistical behaviour of the Favre-averaged scalar dissipation rate conditional on these flow topologies has been analysed in detail for different choices of reaction progress variable. The qualitative behaviour of the scalar-turbulence interaction term in the Favre-averaged scalar dissipation rate transport equation has been found to be affected by the regime of combustion, whereas the chemical reaction rate gradient contribution to the scalar dissipation rate transport has been found to be affected by the choice of the reaction progress variable. The topologies, which exist for all values of dilatation rate, contribute significantly to the Favre-averaged scalar dissipation rate transport in premixed turbulent flames for all regimes of combustion. In addition, the flow topologies, which are obtained only for positive values of dilatation rate, contribute significantly to the Favre-averaged scalar dissipation rate transport in the case representing the corrugated flamelets regime combustion. An unstable nodal flow topology, which is representative of a counter-flow configuration, has been found to be a dominant contributor to the Favre-averaged scalar dissipation rate transport for all regimes of combustion irrespective of the choice of reaction progress variable.

Keywords: Scalar dissipation rate, turbulent premixed combustion, flow topology, velocity gradient tensor, combustion regimes 


\section{INTRODUCTION}

Scalar dissipation rate (SDR) plays a pivotal role in turbulent reacting flows as this quantity characterises the rate of micro-mixing [1], and the mean/filtered reaction rate is proportional to the product of mean/filtered gas density $\rho$ and Favre averaged/filtered SDR in the flamelet regime of combustion [2-5]. The instantaneous SDR of reaction progress variable (RPV) $c$ is defined as [1-5]: $N_{c}=D \nabla c . \nabla c$ where $D$ is the diffusivity of RPV. A number of recent analyses concentrated on the statistical behaviours and modelling of SDR and its transport in different combustion regimes [1,5-8], turbulent Reynolds number [9] and global Lewis number [10,11]. However, the effects of flow topologies on the SDR transport in different regimes of combustion are yet to be clearly understood.

All possible turbulent flow topologies can be categorised into 8 generic canonical flow configurations [12,13], and these are obtained depending on the values of first, second and third invariants (i.e. $P, Q$ and $R$, respectively) of the velocity gradient tensor $A_{i j}=\partial u_{i} / \partial x_{j}=S_{i j}+$ $W_{i j}$ where $S_{i j}=0.5\left(A_{i j}+A_{j i}\right)$ and $W_{i j}=0.5\left(A_{i j}-A_{j i}\right)$. The eigenvalues of $A_{i j}$ are $\lambda_{1}, \lambda_{2}$ and $\lambda_{3}$ which are the solutions of the characteristic equation $\lambda^{3}+P \lambda^{2}+Q \lambda+R=0$ with its invariants $P, Q$ and $R[12,13]$ :

$$
\begin{gathered}
P=-\operatorname{tr}\left(A_{i j}\right)=-\left(\lambda_{1}+\lambda_{2}+\lambda_{3}\right)=-S_{i i} \\
Q=0.5\left(P^{2}-S_{i j} S_{i j}+W_{i j} W_{i j}\right)=Q_{S}+W_{i j} W_{i j} / 2 \\
R=\left(-P^{3}+3 P Q-S_{i j} S_{j k} S_{k i}-3 W_{i j} W_{j k} S_{k i}\right) / 3
\end{gathered}
$$

The positive (negative) values of the discriminant, $D=\left[27 R^{2}+\left(4 P^{3}-18 P Q\right) R+4 Q^{3}-\right.$ $\left.P^{2} Q^{2}\right] / 108$, of the characteristic equation $\lambda^{3}+P \lambda^{2}+Q \lambda+R=0$ lead to focal (nodal) topologies $[12,13]$. The $A_{i j}$ tensor exhibits one real eigenvalue and two complex conjugate eigenvalues for focal topologies, whereas $A_{i j}$ shows three real eigenvalues for nodal topologies. The surface $D=0$ leads to two subsets [12,13]: $r_{1 a}=P\left(Q-2 P^{2} / 9\right) / 3-$ 
$2\left(-3 Q+P^{2}\right)^{3 / 2} / 27$ and $r_{1 b}=P\left(Q-2 P^{2} / 9\right) / 3+2\left(-3 Q+P^{2}\right)^{3 / 2} / 27$. The strain rate tensor $A_{i j}$ has purely imaginary eigenvalues on the surface $r_{2}: R=P Q$ in the region $D>0$. The surfaces $r_{1 a}, r_{1 b}$ and $r_{2}$ divide the $P-Q-R$ phase space into 8 flow topologies (S1-S8), as shown schematically in Fig. 1 [12,13] (S2 topology is schematically shown for later discussion). Relatively limited effort has been directed to the analysis of flow topology distributions in turbulent combustion [14-21]. This analysis considers a detailed chemistry Direct Numerical Simulation (DNS) database of turbulent premixed $\mathrm{H}_{2}$-air flames spanning the corrugated flamelets $(\mathrm{CF})$, thin reaction zones (TRZ) and broken reaction zones (BRZ) regimes. The objectives of this analysis are: (a) to demonstrate the differences in the SDR transport conditional on flow topologies for different combustion regimes; (b) to identify the dominant flow topologies contributing to the SDR transport and offer physical explanations for the observed behaviours. The main implications of the aforementioned objectives are to identify dominant flow topologies which contribute to the SDR transport so that simplified experimental and theoretical frameworks can be constructed based on simplified canonical configurations.

\section{MATHEMATICAL BACKGROUND \& NUMERICAL IMPLEMENTATION}

The RPV $c$ can be defined as: $c=\left(Y_{0}-Y\right) /\left(Y_{0}-Y_{\infty}\right)$ where $Y$ denotes the mass fraction of the species used for the definition of RPV, and the subscripts 0 and $\infty$ depict values in the unburned and fully burned gases, respectively. The transport equation of the Favre-averaged SDR $\widetilde{N}_{c}=\overline{\rho D \nabla c \cdot \nabla c} / \bar{\rho}$ takes the following form after ignoring the contributions of $D$ variations $[1-11]$ :

$$
\bar{\rho} \frac{\partial \widetilde{N_{c}}}{\partial t}+\bar{\rho} \tilde{u_{\jmath}} \frac{\partial \widetilde{N_{c}}}{\partial x_{j}}=\underbrace{\frac{\partial}{\partial x_{j}} \overline{\left[\rho D \frac{\partial N_{c}}{\partial x_{j}}\right]}}_{D_{1}}+T_{1}+T_{2}+T_{3}+T_{4}-D_{2}
$$




$$
\begin{gathered}
T_{1}=-\partial\left(\overline{\rho u_{\jmath}^{\prime \prime} N_{c}^{\prime \prime}}\right) / \partial x_{j} \\
T_{2}=-2 \overline{D[\dot{w}+\nabla \cdot(\rho D \nabla c)]\left(\partial c / \partial x_{k}\right)\left(\partial \rho / \partial x_{k}\right) / \rho} \\
\left.T_{3}=-\overline{2 \rho D\left(\partial c / \partial x_{\jmath}\right)\left(\partial u_{\jmath} / \partial x_{k}\right)\left(\partial c / \partial x_{k}\right.}\right) \\
T_{4}=2 \overline{D\left(\partial \dot{w} / \partial x_{k}\right)\left(\partial c / \partial x_{k}\right)} \\
\left.D_{2}=2 \overline{\rho D^{2}\left(\partial^{2} c / \partial x_{k} \partial x_{l}\right)\left(\partial^{2} c / \partial x_{k} \partial x_{l}\right.}\right)
\end{gathered}
$$

where $\bar{q}, \tilde{q}=\overline{\rho q} / \bar{\rho}$ and $q^{\prime \prime}=q-\tilde{q}$ are the Reynolds average, Favre-average and Favre fluctuation of a general variable $q$ respectively, and $\dot{w}$ is the chemical reaction rate. In eq. $2 \mathrm{i}$, $D_{1}$ is the molecular diffusion term and $T_{1}$ is the turbulent transport term. The terms $T_{2}, T_{3}, T_{4}$ and $\left(-D_{2}\right)$ are referred to as the density gradient, scalar-turbulence interaction, reaction rate gradient and molecular dissipation contributions to the SDR transport.

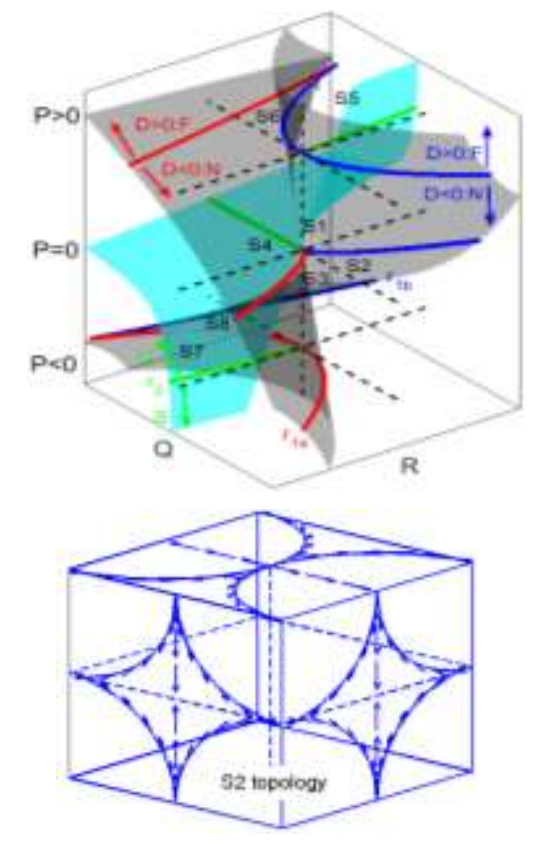

Figure 1: (Top) Classification of $\mathrm{S} 1-\mathrm{S} 8$ topologies in the $P-Q-R$ space. The lines (defined by the cyan and grey surfaces) $r_{1 a}$ (red), $r_{1 b}$ (blue) and $r_{2}$ (green) dividing the topologies are shown. Black dashed lines correspond to $Q=0$ and $=0$. (Bottom) Schematic of S2 topology: unstable node and 2 saddle points.

The statistical behaviours of the unclosed terms $T_{1}, T_{2}, T_{3}, T_{4}$ and $\left(-D_{2}\right)$ conditional on flow topologies will be analysed here based on a three-dimensional DNS [22] database, employing 
a detailed chemical mechanism [20] with 9 steps and 19 reactions for hydrogen-air flames. The unburned gas temperature $T_{0}$ is taken to be $300 \mathrm{~K}$, which gives rise to an unstrained laminar burning velocity $S_{L}=135.6 \mathrm{~cm} / \mathrm{s}$ under atmospheric pressure. Details are found in Ref. [22] and here a brief description is provided. Turbulent inflow and outflow boundaries are specified in the direction of mean flame propagation and transverse boundaries are periodic. The mean inlet velocity has been modified gradually to match the turbulent flame speed as the simulation progresses so that a statistically stationary state can be obtained. The inflow values of normalised root-mean-square turbulent velocity fluctuation $u^{\prime} / S_{L}$, turbulent length scale to flame thickness ratio $l_{T} / \delta_{t h}$, Damköhler number $D a=l_{T} S_{L} / u^{\prime} \delta_{t h}$, Karlovitz number $K a=$ $\left(\rho_{0} S_{L} \delta_{t h} / \mu_{0}\right)^{0.5}\left(u^{\prime} / S_{L}\right)^{1.5}\left(l_{T} / \delta_{t h}\right)^{-0.5}$ and turbulent Reynolds number $R e_{t}=\rho_{0} u^{\prime} l_{T} / \mu_{0}$ for all cases are listed in Table 1 where $\rho_{0}$ is the unburned gas density, $\mu_{0}$ is the unburned gas viscosity, $\delta_{t h}=\left(T_{a d}-T_{0}\right) / \max |\nabla T|_{L}$ is the thermal flame thickness and the subscript ' $\mathrm{L}$ ' is used to refer to the unstrained laminar flame quantities. The instantaneous, adiabatic flame and unburned gas temperatures are shown by $T, T_{a d}$ and $T_{0}$ respectively. The cases investigated here are representative of the $\mathrm{CF}$ (i.e. case A with $K a<1$ ), TRZ (i.e. case B with $1<K a<$ 100 ) and BRZ (i.e. case $\mathrm{C}$ with $\mathrm{Ka}>100$ ) regimes [23].

Table 1:

\section{List of inflow turbulence parameters}

\begin{tabular}{|c|c|c|c|c|c|}
\hline Case & $u^{\prime} / S_{L}$ & $l_{T} / \delta_{t h}$ & $R e_{t}$ & $D a$ & $K a$ \\
\hline A & 0.7 & 14.0 & 227 & 20.0 & 0.75 \\
\hline B & 5 & 14.0 & 1623 & 2.8 & 14.4 \\
\hline C & 14 & 4.0 & 1298 & 0.29 & 126 \\
\hline
\end{tabular}


For cases A and B (case C) the domain size is taken to be $20 \mathrm{~mm} \times 10 \mathrm{~mm} \times 10 \mathrm{~mm}(8 \mathrm{~mm} \times$ $2 \mathrm{~mm} \times 2 \mathrm{~mm}$ ), which has been discretised by a uniform Cartesian grid of dimension $512 \times$ $256 \times 256(1280 \times 320 \times 320)$. The simulation time corresponds to $1.0 l_{T} / u^{\prime}, 6.8 l_{T} / u^{\prime}$ and $6.7 l_{T} / u^{\prime}$ for cases A-C respectively, and is comparable to several previous analyses $[15,16,24-$ 27]. All the statistics are presented in the paper for the aforementioned time instants. However, the statistics shown in the paper remained qualitatively the same since halfway through the simulation.

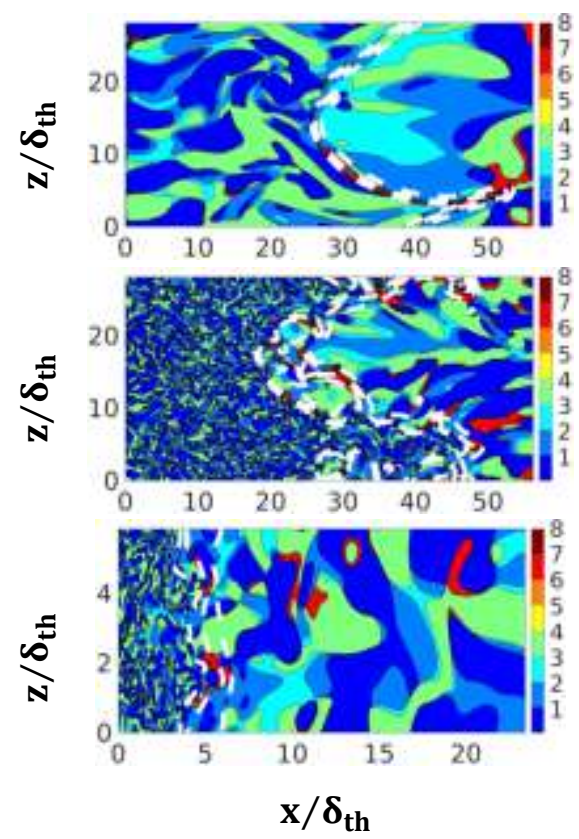

Figure 2: Instantaneous local flow topology fields: (top to bottom) cases A-C. Thick white lines show contours of $c=0.1,0.5,0.9$ (left to right) based on $H_{2}$ mass fraction.

\section{RESULTS \& DISCUSSION}

The spatial distribution of flow topologies and contours of $H_{2}$ based RPV for $c=0.1,0.5$ and 0.9 (from left to right) are shown in Fig. 2, which indicates that the flow topology distributions are different between cases A-C. The flow is dominated, in order, by topologies $\mathrm{S} 1, \mathrm{~S} 4, \mathrm{~S} 2, \mathrm{~S} 3$, altogether covering $95 \%$ by volume of the flow field. A more quantitative evaluation for the flame region $(0.05 \leq c \leq 0.95)$ is shown in Fig. 3 where the volume size distribution of connected topology regions, identified based on a flood fill algorithm, is shown. The integral 
volume of S1-S4 topologies amounts to approximately $84 \pm 1 \%$ but they remain dominant contributors within the flame because they appear for all values of $P$ (see Fig. 1). It has been found that the occurrence of topologies $\mathrm{S} 1, \mathrm{~S} 3, \mathrm{~S} 4$ decreases within the flame, whereas the occurrence of S2,S7 increases in all cases and this redistribution is increasingly less pronounced going from case A to case C. These trends can be qualitatively observed from Fig. 2 and exemplarily it is mentioned that the integral volume for topology S2 (S4) increases in the flame by $9.6,5.5,2.6 \%(8.8,10.0,1.25 \%)$ for cases $\mathrm{A}$ to $\mathrm{C}$ respectively, relative to the distribution in the whole domain. The frequent occurrence of the nodal S2 topology within the flame is also consistent with previous findings for statistically planar premixed flames [16,17], flame-droplet interaction [18] and autoigniting turbulent shear flows [21]. The dilatation rate is predominantly positive within the flame and thus S5 and S6, which are typical of $P=(-\nabla \cdot \vec{u})>0$, are rarely obtained there. The relative strength of dilatation rate and likelihood of obtaining high positive values of $\nabla \cdot \vec{u}$ are significantly smaller in case $C$ than in cases A and B because energetic turbulent eddies penetrate into the reaction zone and disturb the chemical reaction, which affects the magnitude of $\nabla \cdot \vec{u}$ [19]. However, previous analyses indicated that the magnitude of heat release rate does not necessarily decrease with increasing Karlovitz number but the heat release rate becomes less significant in comparison to turbulent transport contributions [28,29]. The weakening of $\nabla \cdot \vec{u}$ in case $\mathrm{C}$ is reflected in almost disappearance of the $\mathrm{S} 8$ topology which covers for case $\mathrm{C}$ less than $0.1 \%$ volume percent of the region $0.05<\tilde{c}<0.95$ (note the logarithmic distribution in Fig. 3). Case B shows larger range of structures, especially more small structures, than in case $\mathrm{A}$ because $R e_{t}$ in case $\mathrm{B}$ is greater than case $\mathrm{A}$, whereas the value of $l_{T}$ remains the same. In case $\mathrm{C}, l_{T}$ remains smaller which, along with higher $R e_{t}$, leads to considerably smaller structures compared to cases A and B. It is worth noting that, apart from one large connected area on the burned gas side, S2 topologies are, specifically for cases A and B, characterised by relatively small structures (see Figs. 2,3). 


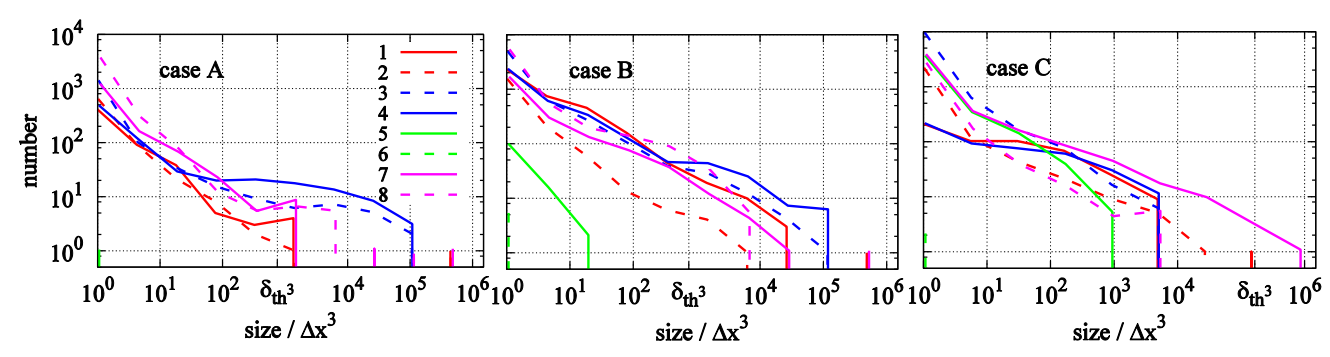

Figure 3: Double logarithmic volumetric size distributions of connected topology regions within the flame $(0.05<\tilde{c}<0.95)$ for cases A-C. The binning size is exponential and the topology size is normalised with the cell volume. For reference the volume $\delta_{t h}^{3}$ is shown on the $\mathrm{x}$-axis. Focal topologies S1, S4, S5, S7 (red-blue-green-magenta solid lines) and nodal topologies S2, S3, S6, S8 (red-blue-green-magenta dashed lines). The same colour scheme is used for Figs. 4, 6 and 7.
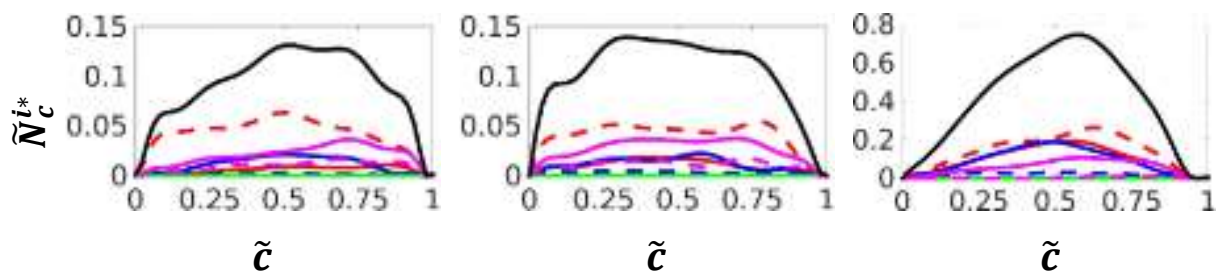

Figure 4: Variation with $\tilde{c}$ of $\widetilde{N}_{c}^{i *}=\widetilde{N}_{c}^{i} \times \delta_{t h} / S_{L}$ where $i=0$ indicates the total $\widetilde{N}_{c}^{*}$ (black solid lines) and $\{i=1, \ldots, 8\}$ are the percentage-topology-weighted values corresponding to S1-8, respectively, for cases (left to right) A-C for $\mathrm{H}_{2}$ based RPV.

The variations of $\widetilde{N}_{c}^{*}=\widetilde{N}_{c} \times \delta_{t h} / S_{L}$ with $\tilde{c}$ for $H_{2}$ based RPV are shown in Fig. 4 (results for $\mathrm{O}_{2}$ and $\mathrm{H}_{2} \mathrm{O}$ based RPVs are not shown because of qualitative similarity with the results for $\mathrm{H}_{2}$ based RPVs) where the contributions conditional upon each topology $\widetilde{N}_{c}^{i *}$ (i.e. $i=1,2, \ldots, 8$ for S1-S8 respectively and $\widetilde{N}_{c}^{*}=\widetilde{N}_{c}^{0 *}=\sum_{i=1}^{8} \widetilde{N}_{c}^{i *}$ ) are also shown. The magnitude of $\widetilde{N}_{c}^{*}$ for a given definition of RPV increases with increasing $u^{\prime} / S_{L}$. For a given case, the peak value of $\widetilde{N}_{c}^{*}$ has been found to be the highest for the $H_{2}$ based RPV and the magnitudes of $\widetilde{N}_{c}^{*}$ remain comparable for $\mathrm{O}_{2}$ and $\mathrm{H}_{2} \mathrm{O}$ based RPVs (not shown here).

Figure 4 shows that S2 remains the major contributor to $\widetilde{N}_{c}$ for cases A and B. However, S7, S4, S8 and S1 (in decreasing order of significance) also contribute to $\widetilde{N}_{c}$ in case A. In case B, S2, S7, S4, S1 and S8 remain the leading contributors in decreasing order of significance. The 
major contributors to $\widetilde{N}_{c}$ are S2, S1, S4 and S7 in case C for all definitions of RPVs. Note that the Favre-averaged SDR $\widetilde{N}_{T}=\overline{\rho \alpha_{T} \nabla c_{T} . \nabla c_{T}} / \bar{\rho}$ of non-dimensional temperature $c_{T}=(T-$ $\left.T_{0}\right) /\left(T_{a d}-T_{0}\right)$ also shows qualitatively similar behaviour as those of $\widetilde{N}_{c}$ and thus is not shown here.
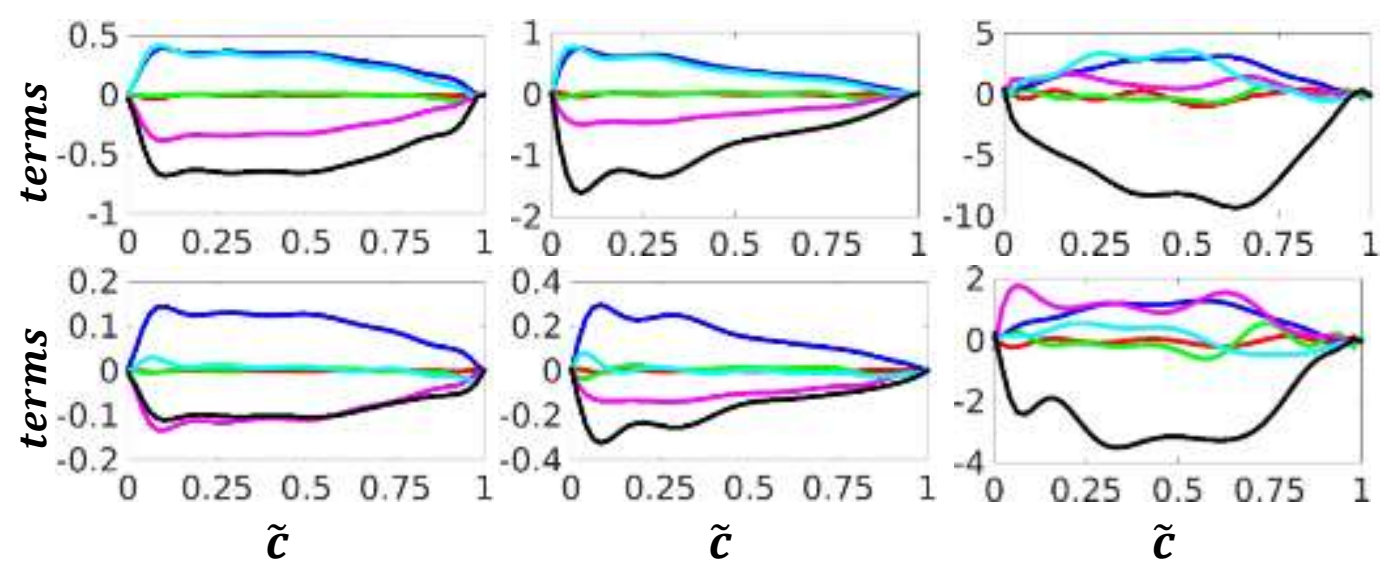

Figure 5: Variation of the $\widetilde{N}_{c}$ transport equation terms for cases (left to right) A-C: $D_{1}$ (red), $T_{1}$ (green), $T_{2}$ (blue), $T_{3}$ (magenta), $T_{4}$ (cyan) and $\left(-D_{2}\right)$ (black) against $\tilde{c}$ for (top to bottom) $\mathrm{H}_{2}$ and $\mathrm{H}_{2} \mathrm{O}$ based RPVs.

The variations of normalised values of $D_{1}, T_{1}, T_{2}, T_{3}, T_{4}$ and $\left(-D_{2}\right)$ with $\tilde{c}$ for $H_{2}$ and $H_{2} O$ based RPVs are presented in Fig. 5 (results for $\mathrm{O}_{2}$ based RPVs are not shown because of qualitative similarity with the results for $\mathrm{H}_{2} \mathrm{O}$ based RPV), which shows the magnitude of the leading contributors increases from case A to case C. Figure 5 shows that $\left(-D_{2}\right)$ acts as a major sink for all cases for all definitions of RPV, and $T_{2}$ acts as a primary source term. The magnitudes of $D_{1}$ and $T_{1}$ remain negligible in comparison to those for $T_{2}$ and $\left(-D_{2}\right)$ in all cases irrespective of the definition of RPV. The term $T_{3}$ acts as an additional important sink for cases $\mathrm{A}$ and $\mathrm{B}$, with its magnitude comparable to that of $\left(-D_{2}\right)$ in case A for $\mathrm{O}_{2}$ and $\mathrm{H}_{2} \mathrm{O}$ based RPVs. The higher molecular diffusivity of $\mathrm{H}_{2}$ than $\mathrm{O}_{2}$ and $\mathrm{H}_{2} \mathrm{O}$ causes $\left(-\mathrm{D}_{2}\right)$ to have higher magnitudes than $T_{3}$ in the case of $H_{2}$ based RPV in case A. In contrast, $T_{3}$ switches from a sink to a major source for case $\mathrm{C}$ and this behaviour is particularly prominent for $\mathrm{O}_{2}$ and $\mathrm{H}_{2} \mathrm{O}$ based RPVs. 

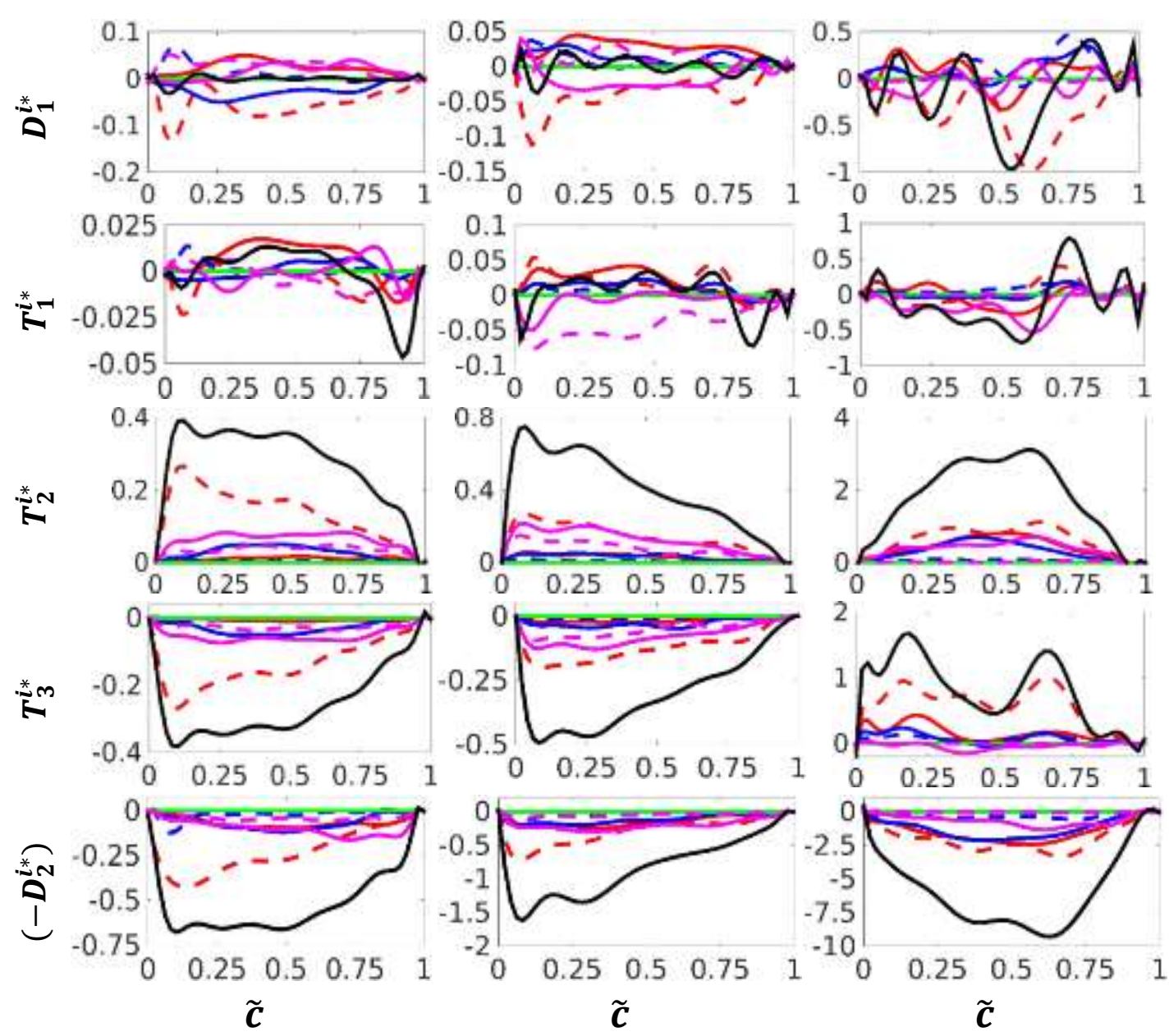

Figure 6: Variation with $\tilde{c}$ of $\left\{D_{1}^{i *}, T_{1}^{i *}, T_{2}^{i *}, T_{3}^{i *}\right.$ and $\left.\left(-D_{2}^{i *}\right)\right\}=\left\{D_{1}^{i}, T_{1}^{i}, T_{2}^{i}, T_{3}^{i}\right.$ and $\left.\left(-D_{2}^{i}\right)\right\} \times$ $\delta_{t h}^{2} / \rho_{0} S_{L}^{2}$ where $i=0$ indicates the total term (black solid lines) and $\{i=1, \ldots, 8\}$ are the percentage-topology-weighted terms corresponding to S1-S8, respectively, for cases (left to right) A-C for $\mathrm{H}_{2}$ based RPV.

The contribution of $T_{3}$ can be expressed as $[6,7,30]: T_{3}=$ $-\overline{2 \rho\left(e_{\alpha} \cos ^{2} \alpha+e_{\beta} \cos ^{2} \beta+e_{\gamma} \cos ^{2} \gamma\right) N_{c}}$ where $e_{\alpha}, e_{\beta}$ and $e_{\gamma}$ are the most extensive, intermediate and the most compressive principal strain rates and the angles of $\nabla c$ with the eigenvectors corresponding to $e_{\alpha}, e_{\beta}$ and $e_{\gamma}$ are given by $\alpha, \beta$ and $\gamma$ respectively. This suggests that a preferential collinear alignment of $\nabla c$ with $e_{\alpha}\left(e_{\gamma}\right)$ results in a positive (negative) value of $T_{3}$. Previous studies $[6,7,30]$ found that $\nabla c$ preferentially aligns with $e_{\alpha}$ when the strain rate induced by flame normal acceleration $a_{\text {chem }}$ dominates over turbulent straining $a_{\text {turb }}$, and a preferential alignment between $e_{\gamma}$ and $\nabla c$ is obtained when $a_{t u r b}$ overcomes $a_{c h e m}$. It was also 
reported $[1,6,7]$ that $a_{c h e m} / a_{\text {turb }}$ can be taken to scale as $a_{\text {chem }} / a_{\text {turb }} \sim\left(T_{a d}-T_{0}\right) f(K a) D a /$ $T_{0}$ where $f(K a)$ is expected to decrease with increasing $K a$. The value of $\left(T_{a d}-\right.$ $\left.T_{0}\right) f(K a) D a / T_{0}$ decreases from cases A to C, which leads to a greater (smaller) extent of $\nabla c$ alignment with $e_{\gamma}\left(e_{\alpha}\right)$ in case $\mathrm{B}$ (case $\mathrm{C}$ ) than in case $\mathrm{A}$ (case $\mathrm{B}$ ). This leads to a preferential alignment of $\nabla c$ with $e_{\alpha}$ for cases $\mathrm{A}$ and $\mathrm{B}$ because of $D a \gg 1$, whereas $\nabla c$ aligns with the most compressive principal strain rate in case $\mathrm{C}$ (where $D a<1$ ). This alignment gives rise to negative (positive) contributions of $T_{3}$ in cases A and B (case C). A previous study [10] showed that a decrease in Lewis number of the RPV leads to an increased (reduced) propensity for $\nabla c$ alignment with $e_{\alpha}\left(e_{\gamma}\right)$, and this leads to a smaller positive contribution of $T_{3}$ in case $\mathrm{C}$ for $\mathrm{H}_{2}$ based RPV than in cases of $\mathrm{O}_{2}$ and $\mathrm{H}_{2} \mathrm{O}$ based RPVs.

Figure 5 also shows that the behaviour of $T_{4}$ changes significantly with the definition of the RPV. In the case of $H_{2}$ based RPV, the term $T_{4}$ acts as a leading source term whereas this term remains negligible in comparison to the magnitudes of $\mathrm{T}_{2}$ and $\left(-\mathrm{D}_{2}\right)$ for $\mathrm{O}_{2}$ and $\mathrm{H}_{2} \mathrm{O}$ based RPVs. On the other hand, $T_{4}$ acts as a leading sink if the RPV is defined based on the nondimensional temperature (not shown here). The term $T_{4}$ can alternatively be expressed as: $T_{4}=$ $-\overline{2 D(\partial \dot{w} / \partial n)|\nabla c|}$ where $n$ is the flame normal direction and $\vec{N}=-\nabla c /|\nabla c|$ is the flame normal vector. Note that $(\partial \dot{w} / \partial n)$ assumes negative (positive) value on the unburned (burned) gas side of the flame. For $\mathrm{H}_{2}$, the distribution of $\dot{w}$ is such that the negative contribution of $(\partial \dot{w} / \partial n)$ is higher in magnitude than its positive contribution and the transition from negative to positive value of $(\partial \dot{w} / \partial n)$ takes place for a $c$ value which is biased towards the burned gas side of the flame front and the opposite is valid for $c_{T}$. This leads to predominantly positive (negative) values of $T_{4}$ for $H_{2}$ (temperature) based RPV. The positive and negative contributions of $(\partial \dot{w} / \partial n)$ are comparable for the case of $\mathrm{O}_{2}$ and $\mathrm{H}_{2} \mathrm{O}$ based RPVs and the transition from negative to positive value takes place close to $c \approx 0.5$, and thus, on averaging 
$-2 D(\partial \dot{w} / \partial n)|\nabla c|$, one obtains a positive (negative) contribution of $T_{4}$ with negligible magnitude towards the unburned (burned) gas side of the flame brush in all cases.
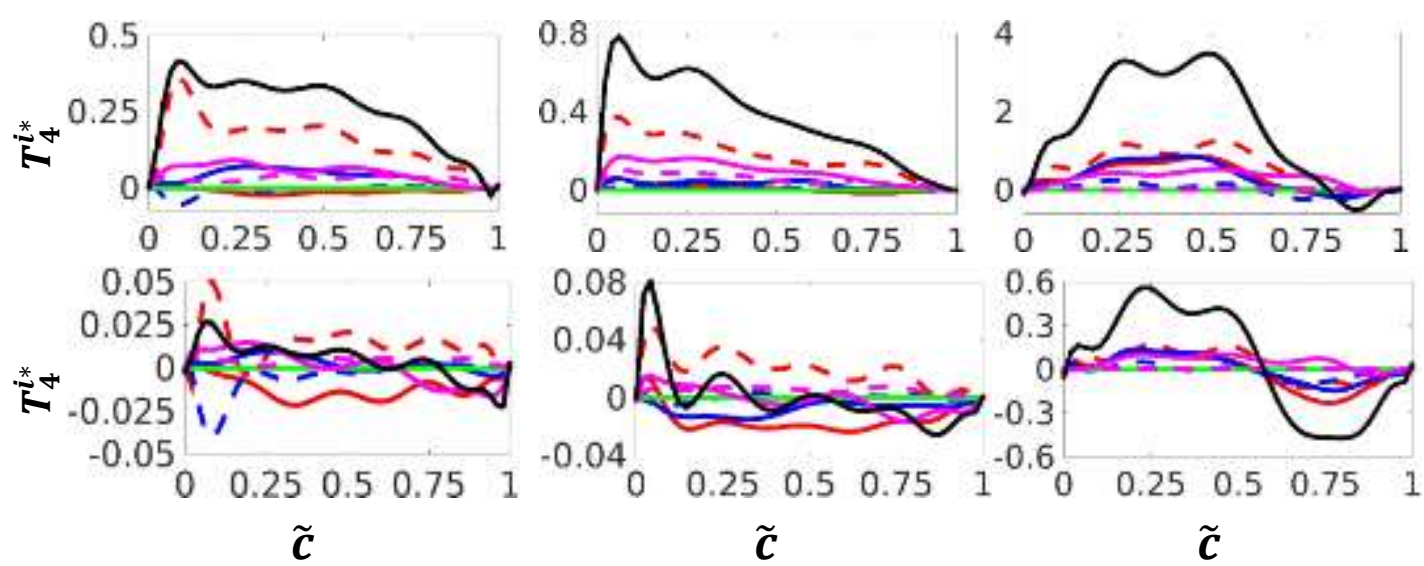

Figure 7: Variation with $\tilde{c}$ of $T_{4}^{i *}=T_{4}^{i} \times \delta_{t h}^{2} / \rho_{0} S_{L}^{2}$ where $i=0$ indicates the total $T_{4}$ (black solid lines) and $\{i=1, \ldots, 8\}$ are the percentage-topology-weighted term corresponding to S18 , respectively, for cases (left to right) A-C for (top to bottom) $\mathrm{H}_{2}$ and $\mathrm{H}_{2} \mathrm{O}$ based RPVs.

The term-by-term contributions of each individual topology to the $\widetilde{N}_{c}$ transport (i.e. $D_{1}^{i}, T_{1}^{i}, T_{2}^{i}, T_{3}^{i}$ and $\left(-D_{2}^{i}\right)$ where $i=1,2, \ldots, 8$ for $S 1-S 8$ such that $T_{j}=T_{j}^{0}=\sum_{i=1}^{8} T_{j}^{i}$ for $j=$ $1-3, D_{1}=D_{1}^{0}=\sum_{i=1}^{8} D_{1}^{i}$ and $\left.-D_{2}=-D_{2}^{0}=-\sum_{i=1}^{8} D_{2}^{i}\right)$ as a function of $\tilde{c}$ based on $H_{2}$ are shown in Fig. 6 for cases A-C. The results for non-dimensional temperature as well as $O_{2}$ and $\mathrm{H}_{2} \mathrm{O}$ based RPVs are not explicitly shown because of qualitative similarities with the $\mathrm{H}_{2}$ based RPV.

It was found that $T_{4}$ is most sensitive to the choices of RPV, and the contributions of each individual topology to $T_{4}$ (i.e. $T_{4}^{i}$ where $i=1,2, \ldots, 8$ for $S 1-S 8$ such that $T_{4}=T_{4}^{0}=$ $\sum_{i=1}^{8} T_{4}^{i}$ ) are shown in Fig. 7 for the RPVs based on $\mathrm{H}_{2}$ and $\mathrm{H}_{2} \mathrm{O}$ (results for $\mathrm{O}_{2}$ based RPV are not shown because of qualitative similarity with the results for $\mathrm{H}_{2} \mathrm{O}$ based RPV). The contributions of flow topologies to $T_{4}$ for $\widetilde{N}_{T}$ are qualitatively similar to those observed for $\mathrm{O}_{2}$ and $\mathrm{H}_{2} \mathrm{O}$ based RPVs and thus are not shown here. 
Table 2

Leading contributions of flow topologies to the different terms of $\widetilde{N}_{c}$ transport.

\begin{tabular}{|c|c|c|c|c|c|c|c|}
\hline Case & $D_{1}$ & $T_{1}$ & $T_{2}$ & $T_{3}$ & $T_{4}\left(H_{2}\right)$ & $\mathrm{T}_{4}\left(\mathrm{H}_{2} \mathrm{O}\right)$ & $-D_{2}$ \\
\hline A & $\mathrm{S} 1-\mathrm{S} 4, \mathrm{~S} 7, \mathrm{~S} 8$ & S1, S2, S4, S7,S8 & $\mathrm{S} 2, \mathrm{~S} 7, \mathrm{~S} 8$ & $\mathrm{~S} 2, \mathrm{~S} 7, \mathrm{~S} 8$ & $\mathrm{~S} 2, \mathrm{~S} 7, \mathrm{~S} 4$ & $\mathrm{~S} 2, \mathrm{~S} 8, \mathrm{~S} 1, \mathrm{~S} 3$ & $\mathrm{~S} 2, \mathrm{~S} 7, \mathrm{~S} 1, \mathrm{~S} 4$ \\
\hline B & $\mathrm{S} 1-\mathrm{S} 4, \mathrm{S7}, \mathrm{S} 8$ & S1, S2, S4, S7,S8 & $\mathrm{S} 2, \mathrm{~S} 7, \mathrm{~S} 8$ & $\mathrm{~S} 2, \mathrm{~S} 7, \mathrm{~S} 8$ & S2,S4,S7 & $\mathrm{S} 2, \mathrm{~S} 8, \mathrm{~S} 1, \mathrm{~S} 4$ & S2,S7, S1, S4 \\
\hline C & S1-S4 & $\mathrm{S} 2, \mathrm{~S} 1, \mathrm{~S} 4, \mathrm{~S} 7$ & $\mathrm{~S} 2, \mathrm{~S} 4, \mathrm{~S} 1$ & $\mathrm{~S} 2, \mathrm{~S} 1, \mathrm{~S} 4, \mathrm{~S} 3$ & $\mathrm{~S} 2, \mathrm{~S} 1, \mathrm{~S} 4, \mathrm{~S} 7$ & S1-S4,S7 & $\mathrm{S} 2, \mathrm{~S} 1, \mathrm{~S} 4, \mathrm{~S} 7$ \\
\hline
\end{tabular}

From Figs. 6 and 7, the topologies which are significant contributors to $D_{1}, T_{1}, T_{2}, T_{3}, T_{4}$ (for $\mathrm{H}_{2}$ and $\mathrm{H}_{2} \mathrm{O}$ based RPVs) and $\left(-D_{2}\right)$ in cases A-C are summarised in Table 2 in the decreasing order of significance. As topology contributions to $T_{4}$ for $O_{2}$ based RPV are similar to that in the case of $\mathrm{H}_{2} \mathrm{O}$ based RPV, this information is not explicitly provided in Table 2. It is evident from Table 2 that S1-S4, S7 and S8 topologies play key roles in the $\widetilde{N}_{c}$ transport in the CF regime flame, but the contributions of S7 and S8 topologies, which are obtained only for positive dilatation rates, weaken with an increase in Karlovitz number, and ultimately for the BRZ regime the $\widetilde{N}_{c}$ transport is principally governed by S1-S4 topologies, although S7 still shows a non-negligible ( $4^{\text {th }}$ important) role for some terms $\left(T_{1}, T_{4}, D_{2}\right)$. The implications in turbulent combustion modelling is that, in the CF regime it is necessary to properly characterise the features of S7, S8 and S1-S4 for accurate prediction of the $\widetilde{N}_{c}$ transport, whereas in the BRZ regime a simple configuration with predominant features of S1-S4 may be sufficient. Furthermore, Table 2 reveals that S2 is the leading contributor to all the terms of the $\widetilde{N}_{c}$ transport equation and thus unstable nodal topologies with saddle points are of key importance for the $\widetilde{N}_{c}$ transport. The counter-flow configuration has the predominant feature of S2, and thus simplified experiments and computations for the flames in this configuration can be an ideal starting point for the analysis of the statistical behaviour and the modelling of the $\widetilde{N}_{c}$ transport. 


\section{CONCLUSIONS}

The Favre-averaged SDR $\widetilde{N}_{c}$ and its transport conditional on flow topologies have been analysed based on a DNS database containing three freely propagating statistically planar $\mathrm{H}_{2-}$ air flames with an equivalence ratio of 0.7 representing the $\mathrm{CF}$, TRZ and BRZ regimes of turbulent premixed combustion. The transport of $\widetilde{N}_{c}$ has been analysed for RPVs based on $\mathrm{H}_{2}, \mathrm{O}_{2}$ and $\mathrm{H}_{2} \mathrm{O}$ mass fractions. It has been found that the weakening of heat release with increasing Karlovitz number affects flow topology distribution and the behaviour of the reacting scalar gradient alignment with local principal strain rates, which in turn affect the nature of the scalar-turbulence interaction term in the $\widetilde{N}_{c}$ transport. Furthermore, the choice of RPV affects the reaction rate gradient contribution to the $\widetilde{N}_{c}$ transport. The flow topologies S1S4, which can be obtained for all values of dilatation rate $\nabla \cdot \vec{u}$, contribute significantly to the $\widetilde{N}_{c}$ transport in all regimes of premixed turbulent combustion. However, the topologies (i.e. S7 and S8), which are obtained only for positive values of dilatation rate (i.e. $\nabla \cdot \vec{u}=-P>0$ ), also contribute significantly to the $\widetilde{N}_{c}$ transport in the CF regime. It has been found in a previous analysis [20] that the topologies S1-S4 also play key roles in the enstrophy transport in all regimes of combustion, whereas $\mathrm{S} 7$ and $\mathrm{S} 8$ topologies play dominant role only in the CF regime. However, no particular flow topology has been found which remains dominant contributor to all the terms of the enstrophy transport equation, whereas an unstable nodal flow topology with saddle points (i.e. S2), which mimics the counter-flow configuration, turns out to be the major contributor to all the source/sink terms in the $\widetilde{N}_{c}$ transport irrespective of the choice of RPV for all regimes of premixed combustion.

For low Mach number flows, as in the cases considered here, the SDR transport equation for passive scalar mixing becomes fundamentally different from premixed turbulent combustion as two important terms $T_{2}$ and $T_{4}$ disappear for passive scalar transport and $T_{3}$ is governed by 
entirely different physics [6,7]. Furthermore, in low Mach number passive scalar mixing without heat release one only obtains flow topologies S1-S4 and the possibility of obtaining topologies S5-S8 completely disappears. In spite of these differences it was reported in Ref. [17] that the $\mathrm{S} 2$ topology dominates turbulent straining which in turn influences $T_{3}$ and $-D_{2}$, which are the leading order terms in the SDR transport for passive scalar mixing. Thus, the available evidences indicate that counter-flow configuration can be a reasonably representative configuration, which plays a dominant role in the SDR transport and thereby can serve as a test bed for fundamental understanding and modelling of the $\widetilde{N}_{c}$ by using simplified experiments and numerical simulations instead of considering complex burner geometries. However, more investigation in other non-canonical configurations will be necessary to claim the dominant behaviour of the counter-flow configuration in the SDR transport.

\section{ACKNOWLEDGEMENTS}

Part of the work presented in this study was sponsored by competitive research funding from King Abdullah University of Science and Technology (KAUST). The work made use of computational resources at KAUST Supercomputing Laboratory and ARCHER at Engineering and Physical Sciences Research Council (EPSRC). 


\section{REFERENCES}

1. N. Chakraborty, M. Champion, A. Mura, N. Swaminathan, Scalar dissipation rate approach to reaction rate closure, Turbulent premixed flame, (Eds. N. Swaminathan, K.N.C. Bray), Cambridge University Press, $1^{\text {st }}$ Edition, 2011, pp.76-102.

2. K.N.C. Bray, , Turbulent Reacting Flows, Springer Verlag, Berlin Heidelburg, New York, eds. P.A. Libby and F.A. Williams, 1980, p. 115-183.

3. N. Chakraborty, R.S. Cant, Combust. Flame, 158 (2011) 1768-1787.

4. T. Dunstan, Y. Minamoto, N. Chakraborty, N. Swaminathan, Proc. Combust. Inst. 34 (2013) 1193-1201.

5. Y. Gao, N. Chakraborty, N. Swaminathan, Combust. Sci Techol. 186 (2014)1309-1337.

6. N. Chakraborty, N. Swaminathan, Phys. Fluids, 19 (2007) 045103.

7. N. Chakraborty, N. Swaminathan, Phys. Fluids, 19 (2007) 045104.

8. N. Chakraborty, J.W. Rogerson, N. Swaminathan, Phys. Fluids, 20 (2008) 045106.

9. N. Chakraborty, N. Swaminathan, Combust. Sci. Technol., 185 (2013) 676-709.

10. N. Chakraborty, N. Swaminathan, Combust. Sci. Technol., 182 (2010)1201-1240.

11. N. Chakraborty, N. Swaminathan, Flow Turb. Combust., 87 (2011) 261-292.

12. A. Perry, M. Chong, Ann. Rev. Fluid Mech.,19 (1987) 125-155.

13. M. Chong, A. Perry, B. Cantwell, Phys. Fluids, 2 (1990)765-777.

14. M. Tanahashi, M. Fujimura, T. Miyauchi, Proc. Comb. Inst., 28 (2000) 529-535.

15. R. Grout, A. Gruber, C. Yoo, J. Chen, Proc. Comb. Inst., 33 (2011) 1629-1637.

16. L. Cifuentes, C. Dopazo, J. Martin, C. Jimenez, Phys. Fluids, 26 (2014) 065108.

17. L. Cifuentes, Local flow topologies and scalar structures in turbulent combustion, $\mathrm{PhD}$ thesis (2015).

18. D.H. Wacks, N. Chakraborty, Fuel, 184 (2016)922-947. 
19. D.H. Wacks, N. Chakraborty, M. Klein, P.G. Arias, H.G. Im, Phys. Rev. F, 1, (2016) 083401.

20. V. Papapostolou, D. H Wacks, M. Klein, N. Chakraborty, H. G. Im, Scientific Reports, 7(1) (2017) 11545 .

21. A. Krisman, E.R. Hawkes, J. H. Chen, J. Fluid Mech. 824 (2017) 5-41.

22. H.G. Im, P.G. Arias., S. Chaudhuri, H.A. Uranakara., Combust. Sci. Technol., 188 (2016) 1182-1198.

23. N. Peters, Turbulent Combustion, Cambridge University Press, 2000.

24. I. Han, K.Y. Huh, Combust. Flame 152 (2008) 194-205.

25. H. Reddy, J. Abraham, Phys. Fluids, 24 (2012) 105108.

26. C. Pera, S. Chevillard, R. Reveillon, Combust. Flame, 160 (2013) 1020-1032.

27. C. Dopazo, L. Cifuentes, J. Martin, C. Jimenez, Combust. Flame, 162 (2015)1729-1736.

28. P.E. Hamlington, A.Y. Poludnenko, E.S. Oran, Phys. Fluids, 23 (2011) 125111.

29. B. Bobbitt, S. Lapointe, G. Blanquart, Phys. Fluids, 28 (2016) 015101.

30. N. Chakraborty, M. Klein, N. Swaminathan, Proc. Combust. Inst., 32 (2009) 1408-1415. 


\section{FIGURE CAPTIONS}

Figure 1: (Top) Classification of $\mathrm{S} 1-\mathrm{S} 8$ topologies in the $P-Q-R$ space. The lines (defined by the cyan and grey surfaces) $r_{1 a}$ (red), $r_{1 b}$ (blue) and $r_{2}$ (green) dividing the topologies are shown. Black dashed lines correspond to $Q=0$ and $R=0$. (Bottom) Schematic of S2 topology: unstable node and 2 saddle points.

Figure 2: Instantaneous local flow topology fields: (top to bottom) cases A-C. Thick white lines show contours of $c=0.1,0.5,0.9$ (left to right) based on $H_{2}$ mass fraction.

Figure 3: Double logarithmic volumetric size distributions of connected topology regions within the flame $(0.05<\tilde{c}<0.95)$ for cases A-C. The binning size is exponential and the topology size is normalised with the cell volume. For reference the volume $\delta_{t h}^{3}$ is shown on the X-axis. Focal topologies S1, S4, S5, S7 (red-blue-green-magenta solid lines) and nodal topologies S2, S3, S6, S8 (red-blue-green-magenta dashed lines). The same colour scheme is used for Figs. 4, 6 and 7.

Figure 4: Variation with $\tilde{c}$ of $\widetilde{N}_{c}^{i *}=\widetilde{N}_{c}^{i} \times \delta_{t h} / S_{L}$ where $i=0$ indicates the total $\widetilde{N}_{c}^{*}$ (black solid lines) and $\{i=1, \ldots, 8\}$ are the percentage-topology-weighted values corresponding to $\mathrm{S} 1-8$, respectively, for cases (left to right) A-C for $\mathrm{H}_{2}$ based RPV.

Figure 5: Variation of the $\widetilde{N}_{c}$ transport equation terms for cases (left to right) A-C: $D_{1}$ (red), $T_{1}$ (green), $T_{2}$ (blue), $T_{3}$ (magenta), $T_{4}$ (cyan) and $\left(-D_{2}\right)$ (black) against $\tilde{c}$ for (top to bottom) $H_{2}$ and $\mathrm{H}_{2} \mathrm{O}$ based RPVs.

Figure 6: Variation with $\tilde{c}$ of $\left\{D_{1}^{i *}, T_{1}^{i *}, T_{2}^{i *}, T_{3}^{i *}\right.$ and $\left.\left(-D_{2}^{i *}\right)\right\}=\left\{D_{1}^{i}, T_{1}^{i}, T_{2}^{i}, T_{3}^{i}\right.$ and $\left.\left(-D_{2}^{i}\right)\right\} \times$ $\delta_{t h}^{2} / \rho_{0} S_{L}^{2}$ where $i=0$ indicates the total term (black solid lines) and $\{i=1, \ldots, 8\}$ are the percentage-topology-weighted terms corresponding to S1-S8, respectively, for cases (left to right) A-C for $H_{2}$ based RPV. 
Figure 7: Variation with $\tilde{c}$ of $T_{4}^{i *}=T_{4}^{i} \times \delta_{t h}^{2} / \rho_{0} S_{L}^{2}$ where $i=0$ indicates the total $T_{4}$ (black solid lines) and $\{i=1, \ldots, 8\}$ are the percentage-topology-weighted term corresponding to S18, respectively, for cases (left to right) A-C for (top to bottom) $\mathrm{H}_{2}$ and $\mathrm{H}_{2} \mathrm{O}$ based RPVs. 